\title{
Health System Strategies and Adolescent Sexual Health: Systematic review using Rodgers Concept Analysis Framework
}

\author{
Wilfred Njabulo Nunu ( $\nabla$ njabulow@gmail.com ) \\ National University of Science and Technology https://orcid.org/0000-0001-8421-1478 \\ Lufuno Makhado \\ University of Venda \\ Jabu Tsakani Mabunda \\ University of Venda \\ Rachel Tsakani Lebese \\ University of Venda
}

Research article

Keywords: Adolescents, Antecedents, Attributes, Consequences, Health Systems, Sexual Health,

Strategies

Posted Date: February 17th, 2020

DOl: https://doi.org/10.21203/rs.2.23715/v1

License: (9) This work is licensed under a Creative Commons Attribution 4.0 International License.

Read Full License 


\section{Abstract}

Background: Health Systems Strategies have been key in determining Adolescent Sexual Health outcomes. This study therefore aims to review literature on relationship between Health Systems Strategies and Adolescent Sexual Health issues guided by Rodgers evolutionary concept analysis framework. The study further develops a Conceptual Framework that would guide a study that seeks to "develop strategies to facilitate safe sexual practices in adolescents through Integrated Health Systems in selected Districts in Zimbabwe".

Methods:The words Adolescents, Health Systems, Sexual Health and Strategies were used to search for published literature (in English) on Google Scholar, PUBMED, EBSCO and Science Direct. 111 Articles and 04 reports were obtained and their content screened for relevance and 32 articles 01 report were found suitable and relevant and thus were reviewed. Thematic analysis to identify attributes, antecedents and consequences of Health Systems Strategies on Adolescent Sexual Health was done. These findings were then used to inform the development of the Conceptual Framework.

Results: Identified attributes were: contextual, dynamic, engaging and advocating, and inefficient Health Systems in addressing Adolescent Sexual Health. Identified antecedents of these Health System Strategies on Adolescent Sexual Health included; adolescent sexual rights, lack of understanding of what sexual health is, need for integrated Adolescent Sexual Health systems, available resources and type of society. Consequences of these Health Systems Strategies on Adolescent Sexual Health were identified to be; weak Adolescent Sexual Health programs, parallel and fragmented Health Systems, vulnerability and heightened risks for poor health outcomes and challenges in interacting with different ethnic groups and gender.

Conclusions: Adolescents are very vulnerable and need to be protected at all costs. There is need to have comprehensive Health Systems Strategies that would positively influence Adolescent Sexual Health.

\section{Background}

Ensuring safe sexual practices in adolescents is one of the key challenges that have been faced even up to the $21^{\text {st }}$ century $[1,2]$. There have been quite a number of factors that have led to negative Sexual Health (SH) outcomes in adolescents. Different authors have noted these as: unfriendly sexual health care services for adolescents, poor health care financing just to mention a few [2, 3]. An adolescent in this study would be defined as a young person who is in the age group of 10 to 19 years and is in the process of transitioning into adulthood $[4,5]$. Adolescent utilisation of $\mathrm{SH}$ care services in low and middle income countries remains low despite quite a number of strategies that have been implemented to create demand for these services $[1,6]$. Most countries find it very challenging to provide comprehensive and integrated HSs to cater for adolescents' $\mathrm{SH}$ issues $[7,8]$. Demand and utilisation of modern health care services by adolescents still remains low despite this huge investment in these services $[1,6]$. 
Health Systems (HSs) play a major role in ensuring access to SH services by adolescents and in turn impact on their SH outcomes [6]. HSs have been defined as organisation of people, institutions and resources so as to ensure delivery of SH services to adolescents $[4,9,10]$. It is of importance to note that this age group is not totally independent in making their decisions therefore would rely on their environments to inform their decisions [10].

There are quite a number of strategies that have been implemented world-wide so as to improve Adolescent Sexual Health (ASH) outcomes $[1,11,12]$. Strategies in this study will be defined as a plan that is implemented within a HS so as to impact on SH outcomes of adolescents [13]. Despite implementation of quite a number of strategies adolescents still remain highly vulnerable, with high prevalence of Sexually Transmitted Infections (STIS), high incidence of teenage pregnancy resulting in high numbers of adolescents dropping out of schools [3,10,14]. Dropping out of formal schools subjects adolescents to poverty particularly in Low and Middle Income Countries (LMCls) where employment opportunities are hard to come by and there is stiff competition for the few available opportunities [2]. Concepts are not well understood when it comes to implementation of ASH programs leading to low demand of such programs that are meant to improve their SH outcomes $[1,6]$. This study therefore aims to review literature on relationship between HSSs and ASH issues guided by Rodgers evolutionary concept analysis framework. The study further aims to leverage on the findings of this literature review to develop a Conceptual framework (CF) that would guide a research on "Development of strategies to facilitate safe sexual practices in adolescents through integrated health systems in selected districts of Zimbabwe".

\section{Methods}

\subsection{Rodgers Concept analysis framework}

This study would be guided by Rodgers' evolutionary concept analysis framework. Rodgers argued that for one to clearly understand the nature of concepts, there is need to understand the contextual forces that influence that specific concept at a specific time and point $[15,16]$. It should be noted that these concepts are dynamic and evolve with time [15]. Rodgers's CF leverages on three key elements: a) Antecedents- these are classified as events or incidents that must occur prior to the occurrence of the concept; b) Attributes - that are described as characteristics of that specific concept in terms of addressing its intended issue; c) Consequences under this framework are defined as the outcomes of the specific concepts as identified from literature $[17,18]$.

\subsection{Collection and analysis of data}

\subsubsection{Inclusion Criteria}

In this systematic review we considered studies that presented Health System Strategies that targeted Adolescent Sexual Health. We targeted studies and reports that were published in English up to December 2018 in peer reviewed journals world over. We focussed on original quantitative and qualitative research 
and reports obtained from Google Scholar, PUBMED, EBSCO and Science Direct. The selected articles and reports had to pass the quality assessment criteria described in 2.2.6.

\subsubsection{Exclusion Criteria}

Studies that focussed on the relationship between Health System Strategies and their impact on other age groups (other than adolescents) were excluded from this study.

\subsubsection{Search Strategy}

Keywords: Adolescents, Health Systems, Sexual Health and Strategies were used to search for relevant literature from Google Scholar, PUBMED, EBSCO and Science Direct.

\subsubsection{Methods of review}

Titles and abstracts were reviewed independently by all three authors to identify articles and reports that were relevant and qualified to be included in this study. Disagreements were resolved through dialogue between the three authors and reaching a consensus based on facts exchanged. Full texts of these articles and reports that met the inclusion criteria were also reviewed by all three authors and findings discussed and an agreement reached.

\subsubsection{Data Extraction and synthesis}

A data collection form was developed guided by Rodgers Evolutionary Conceptual Analysis Framework to facilitate uniform data collection (by all authors) on attributes, antecedents and consequences of Health System Strategies on Adolescent Sexual Health from the articles and reports that met the inclusion criteria. All the three authors reviewed articles and reports and collected data. Discrepancies observed on collected data by the three authors were resolved through dialogue and reaching a consensus. Findings from the articles and reports were coded and thematically analysed to identify and explain antecedents, attributes and consequences of HSSs on ASH.

\subsubsection{Quality Assessment}

A quality evaluation tool was used to assess the quality of the selected studies in line with Rodgers' Evolutionary Concept Analysis Framework [19]. This tool enables one to determine whether original studies adhered to 14 quality criteria for quantitative studies and 10 point quality criteria for qualitative studies [19]. Articles and reports were assessed for clarity in the presentation of attributes, antecedents and consequences of HSSs on ASH. Furthermore the AMSTAR tool for assessing methodological quality of systematic reviews was used to assess the quality of the methods used in this systematic review (as shown in Table 4). The AMSTAR tool was developed to aide assessment of methodological quality and rigour of systematic reviews conducted by different authors [20]. A PRISMA checklist was also completed to ensure that the study fulfilled the expectations of review articles.

\subsection{Timeline}


Literature search was done from January 2019 to May 2019. Quality appraisal then followed in June 2019 whilst data extraction, synthesis and writing up was done between end of March to July 2019.

\subsection{Development of a conceptual framework}

Findings from this study informed the development of a Conceptual framework that explained the relationship between the identified antecedents and attributes influence adolescents' sexual outcomes (consequences). The theoretical framework would be presented diagrammatically so as to aide understanding of how these factors are interrelated as they relate to adolescent sexual health.

\section{Results}

There were 849 articles and 18 reports that were obtained through electronic search of databases. After screening for duplicates, 646 articles and 16 reports had their titles and abstracts reviewed by all the three authors. Of these reviewed articles and reports, 535 articles and 12 reports were excluded as their content was irrelevant to this study. This meant that 111 articles and 4 reports were reviewed to determine whether or not they met the inclusion criteria. After reviewing the articles and reports the three authors resolved that 32 articles and one report met the inclusion criteria and there were then analysed. These results are summarised in the PRISMA flow diagram on Fig 1. Furthermore a Systematic Review protocol is appended as a supplementary file so as to clearly specific what this review sought out to achieve.

\subsection{Outcome of Quality Assessment Tools}

All selected articles were subjected to the 14 point quality assessment tool and met the minimum standards required. The AMSTAR tool results on Methodological Quality pointed out that the methods used were of sufficient quality to address the research questions of this systematic review. These findings are presented on Table 4.

\subsection{Definition of Sexual Health}

The definition of sexual health has evolved over the years leveraging on the 1975 World Health Organization that defined sexual health as "Integration of the somatic, emotional, intellectual and social aspects of sexual being in ways that are positively enriching and that enhance personality, communication and love" $[14,21,22]$.The change in these definitions have been influenced by political, social, historic events and human right considerations [3, 21]. After the sexual revolution in the 1960s, there has been an on-going struggle over reproductive rights, rights for homosexuals and abortion rights for adolescents [21]. These struggles are influenced by different contextual factors with some countries rejecting implementing or aligned to adopt some of the proposed sexual rights [23, 24].These have influenced the way health systems are structured in different countries with different contextual features resulting in varying adolescent sexual health outcomes.

\subsection{Attributes, Antecedents and Consequences of HSSs in relation to ASH}




\subsubsection{Attributes}

Sexual health attributes that were obtained from literature were: contextual, dynamic, activism and advocacy and inefficient. Literature sources reporting these attributes are summarised on Table 1.

\subsubsection{Contextual}

There is no consensus in how sexual health is defined, interpreted and infused into HSs within different communities in different countries [21,22]. HSSs that are implanted in different communities in different countries are influenced by different contextual factors such as political environment, culture, religion and many more that will be prevailing at that specific time and point $[14,21,22]$. There is also need that the HSSs align and observe different societal values and expectations [25].

\subsubsection{Dynamic}

Different HSSs are implemented to address ASH issues in response to the ever changing environments [26-29]. It is presented that strategies to address sexual health issues have evolved in line with the ever changing environmental and contextual factors leading to utilisation of different technological platforms so as to ensure relevance of the strategies in addressing ASH issues [14, 21, 22, 27, 29].

\subsubsection{Activism and Advocacy}

Most HSSs as identified in literature leverage on activism and advocacy to create demand for ASH programs [30-32]. Different strategies are demonstrated as health service providers utilise different strategies so as to involve adolescents and lure them into programs that target their knowledge, attitudes and behaviours so as to promote safe sexual practices [33-35].

\subsubsection{Inefficient}

Due to differences in adolescents age groups, social status, ethnic beliefs and expectations; designed HSSs are never comprehensive enough [14,21]. It is therefore noted that most HSSs have their strengths and weaknesses, therefore putting some adolescent populations at risk whilst on the other hand giving advantages to other adolescent populations $[25,26,36-38]$.

\subsubsection{Antecedents}

Antecedents that influence ASH that were reported in literature were: adolescent sexual rights, lack of understanding of what sexual health is, need for integrated ASH systems, available resources and type of society. Literature sources reporting these attributes are summarised on Table 2.

\subsubsection{Adolescent Sexual Rights}

One of the antecedent themes that arose from literature was the need to protect adolescents as a human right necessity / requirement $[2,39]$. Authors argue that adolescent sexual rights such as the right to 
access sexual health information are more often violated $[2,39]$. There are arguments that sexual education is restricted in schools or in religious setups thereby denying adolescents key information that would inform their decisions regarding sexual health [14]. Most strategies have therefore been targeted at improving adolescent access to information and different strategies have been used by different health systems to disseminate as much information as possible on sexual health [40]. Some of these strategies have leveraged on available technologies (social media, utilisation of sports just to name a few) to ensure that information is disseminated to adolescents [31]. Authors further argue that the current health systems are rigid and violate adolescents rights [41]. Flicker and Guta (2007) argue that there is need that adolescents be allowed to make their own decision in being part of researches that are meant to gather data that is aimed at improving their sexual health outcomes [41]. They argue that involving parents in consenting to adolescents participating in sexual research silences them and this impacts negatively on the process of collecting data that answers questions regarding the expectations of the adolescents [41].

\subsubsection{Lack of understanding of what sexual health is.}

Sexual health definitions have evolved over the years and have been contextualised to suit certain specific environmental contexts [14, 21]. This has created varied definitions that are sometimes contradictory and create a lot of confusion with different countries contextualising the definitions to suit their contexts [21]. The universal understanding of what sexual health entails is therefore influenced by cultural, social, political and environmental contexts and might differ from country to country or continent to continent $[14,21]$. HSs therefore have been designed in a way that lacks comprehensiveness as there are varied expectations which normally undermine international expectations and recommendations regarding management of ASH issues [14,21]. Some authors argue that most health service providers do not speak about sexual health issues as often as they are expected to so as to proactively address sexual health concerns of adolescent [37]. Lack of such interaction has created huge gaps of knowledge in adolescents $[37,38]$. It is also presented that most studies evaluating the impact of different HSSs on promoting sexual health in young people often lack methodological rigour leading to uncomprehensive conclusions and recommendations that do not fully inform policy makers on the direction that they should take with their strategies if they are to promote safe sexual practices in young people [40].

\subsubsection{Need for integrated ASH systems}

Adolescents need to be exposed to programmes that enhance their skills in negotiating for safe sexual behaviours [42]. The approaches or strategies used should be comprehensive in providing them with skills that enhance their ability to make beneficial relationships (with their parents, guardians and adults in general) that are supportive and offer them guidance towards career prospects that are economically sound [5, 42]. ASH strategies should therefore go beyond sexual issues to provide an integrated platform to ensure wholesome development in these adolescents [5]. Strategies targeting these groups should therefore be driven by the need to facilitate development and enhance skills well beyond sexual health issues and offer equal opportunities for both girls and boys [42]. There is a gap that ASH programs are 
not integrated into main HS structures thereby leading to less time and resources being dedicated to this cause $[11,40]$.

\subsubsection{Available Resources}

HSSs are crafted on the basis of available resources to fund and sustain specific sexual health programs [37]. Health service providers in resource poor settings avoid "opening a can of worms" that is, asking questions or following up on adolescent sexual health issues that would require more resources and time than the HS is able to offer at that specific time and point [37]. Lack of resources leads to non-

prioritisation of adolescent sexual health issues and avoidance of asking or following up on matters that might have a bearing on ASH issues [37, 38].

\subsubsection{Type of society}

HSSs are influenced by different ethnic values [25, 37]. Most people identify with different cultural norms that could be for or against certain HSSs [25]. Strategies that clash with specific societal beliefs are bound not to work. Adolescents from poor settings such as those that are from rural areas are more at risk of suffering negative sexual health outcomes as compared to those in urban areas that have easy access to information and health facilities [26, 36]. Adolescents utilising Indigenous Health Systems (IHSs) often have poor health outcomes as compared to utilising Modernised Health Systems (MHSs) [43]. Crafting and implementation of HSSs to address ASH issues are therefore leveraged on specific societal characteristics so as to generate demand for ASH services [14, 25].

\subsubsection{Consequences}

Consequences of these attributes and antecedents were reported to be: Weak adolescent programs constrained by different factors, parallel or fragmented systems resulting in low impact, vulnerability and heightened risks for poor health outcomes and challenges in interacting with different ethnic groups and gender. Literature sources reporting these consequences are summarised on Table 3.

\subsubsection{Weak adolescent programs constrained by different factors}

Adolescent sexual health programs normally fail to achieve desired health outcomes. There is existence of stigma and controversial attitudes that undermine these adolescent sexual health programs rendering them ineffective [5]. This exposes adolescents to incomprehensive programs that exacerbates their vulnerability particularly fuelling confusion in how adolescents are expected to conduct themselves regarding sexual health issues [5]. Adolescents therefore become victims of sexual violence, early pregnancies, unsafe abortions and STIs $[2,5]$.

\subsubsection{Parallel or fragmented systems resulting in low impact.}

I was revealed that a number of ASH programs are delivered as specific fragments addressing specific programs [11]. Sexual health programs such as those addressing STIs, HIV and AIDS and family planning 
are normally funded by different organisations leading to a fragmented approach in addressing ASH issues [11]. Most HSSs therefore lack comprehensiveness and run parallel to each other though delivered to the same recipients leading to conflicts, duplication, confusion and inefficient utilisation of resources [11].

\subsubsection{Vulnerability and heightened risks for poor health outcomes.}

Adolescents are placed at heightened risks for poor sexual health outcomes due to non-compatibility of HSSs with their developmental needs in relation to their specific age groups [3, 27, 42]. There is need to contextualise strategies in such a way that it takes into consideration the different developmental stages and the needs of these adolescents thereby ensuring that the strategy used is appropriate to attain desired goals [5, 27-29].

\subsubsection{Challenges in interacting with different ethnic groups and gender.}

Authors point out that no matter how accommodating some HSs are to adolescents, some ethnic groups were born from less liberal communities and are not forth coming in discussing sexual health related issues [37]. It is also presented that gender plays an important role as some health service providers prefer discussing sexual related issues with service recipients of the same sex [37,38]. This disadvantages some service recipients as they are denied the chance to be given information that they could pass on to their children in cases of adults thereby disturbing sexual health information dissemination [38]. There are also challenges where most health service providers find it challenging to discuss sexual health issues with homosexuals [37]. These challenges create barriers in HS functions in terms of information dissemination that is expected to foster safe sexual practices in adolescents $[23,24$, 37].

\section{Discussion}

\subsection{Conceptual framework}

The CF based on the findings of this study is presented as Fig 2 as a summarised model. The model would also include proposed strategies found in literature that are aimed at improving ASH outcomes.

\subsection{Proposed Strategies to overcome barriers to Safe Sexual Practices in Adolescents}

\subsubsection{Training}

It is evident from the findings of this study that most HSs are very unfriendly to adolescents. They are often characterised as being judgemental, less tolerant and hostile towards adolescents [7,44]. There is generally proposals from literature that suggest that for HSSs to be effective in addressing ASH issues there is need to train HSPs to be accommodative, sensitive and tolerant to adolescents [37]. This will enable these ASH systems to be accessible to adolescent through generation of demand for services 
offered. This would improve coverage of ASH programs and thus minimise barriers associated with poor relation between adolescents and HSPs.

\subsubsection{Providing Sufficient Information on SH}

Findings in this study suggest that $\mathrm{SH}$ is misunderstood and the definitions are not internationalised. Different contextual factors influence the definitions of $\mathrm{SH}$ and different communities have their own understanding of what sexual health is. Different strategies therefore are used in relation to different population groups. There are proposals that $\mathrm{SH}$ issues should be infused into curriculum at schools so as to ensure that all adolescents get relevant information on $\mathrm{SH}[3,29]$. Outside the school setup, some conservative communities might not permit SH information sharing between parents and their adolescents $[45,46]$. Coming up with strategies that are contextualised to different adolescent age groups would enable effective information sharing and promote safe sexual practices in adolescents [5, $27,29,37]$. There is also need to promote dialogue between adolescents and their parents so as to facilitate information sharing and responsible parenting and guidance in as far as $\mathrm{SH}$ issues are concerned [47].

\subsubsection{Expanding the roles of HSPs to ensure coverage}

It is presented in the findings that HSPs do not address $\mathrm{SH}$ issues proactively with adolescents. This makes the services rendered to be ineffective and disjointed [37]. HSPs should take a leading role in information dissemination and marketing of ASH programs to an extent that their services could be extended to schools $[29,37]$. This would ensure demand generation for ASH programs, trust building between HSPs and adolescent and the general community. This would result in reduction of conflicts between different HSs and fostering an inclusive and integrated approach to management of ASH issues.

\subsubsection{Limitations of this study}

Findings from the different articles and report reviewed were not presented independently but collectively according to themes. This could have led to biases towards themes and not the in-depth and rich findings of each respectively reviewed articles and report study.

\section{Conclusions}

Adolescents are very vulnerable and need to be protected at all costs. There is need to have comprehensive HSSs that would positively influence ASH. This would in turn reduce their vulnerability and ensure they access HSs and utilise them in a manner that would improve their SH outcomes. Providing trainings, information sharing and integrating ASH systems is key in achieving desired ASH outcomes.

\section{Abbreviations}


AIDS Acquired Immunodeficiency Syndrome

ASH Adolescent Sexual Health

CF Conceptual Framework

HIV Human Immunodeficiency Virus

HS Health System

HSP Health Service Provider

HSS Health System Strategy

IHS Indigenous Health System

MHS Modern Health System

SH Sexual Health

STIs Sexually Transmitted Infections

\section{Declarations}

\subsection{Ethics approval and consent to participate}

This study is part of PhD studies at the University of Venda. Permission to conduct this review was granted by the University of Venda ethics Committee (Ethics number: SHS / 19 / PH / 17/2608).

\subsection{Consent for publication}

Not Applicable

\subsection{Availability of data and material}

Not Applicable

\subsection{Competing interests}

The authors declare that they have no competing interests.

\subsection{Funding}

The research would be funded by the National University of Science and Technology under the Staff Development Programme. The funder pays for tuition fees related to these PhD studies. The funder would also provider resources to cover data collection, analysis and remuneration of two data collectors who will assist the principal investigator WNN. Researchers will write and submit six monthly reports to 
appraise the funder of progress. The funder's role is to provide resources to successfully carry out the proposed project.

\subsection{Authors' contributions}

WNN is a PhD in Public Health student at the University of Venda. The author conceptualised the idea and drafted the Systematic review manuscript as partial fulfilment of the requirements of the PhD requirements. LM is the Supervisor of these PhD studies whilst JTM and RTL are Core Supervisors. The three have contributed through guiding the PhD student in the conceptualisation, reviewing of articles and reports and preparation of this systematic review manuscript. All the authors read and approved the final manuscript.

\subsection{Acknowledgements}

Not Applicable

\subsection{Authors' information}

WNN is a PhD in Public Health student at the University of Venda in South Africa, and is also a Lecturer in the Department of Environmental Science at National University of Science and Technology in Bulawayo in Zimbabwe. LM is a Senior Lecturer in the Department of Public Health under the School of Health Sciences at the University of Venda. JTM is a Lecturer in the Department of Public Health under the School of Health Sciences at the University of Venda in Thohoyandou in South Africa. RTL is a Research Professor at the School of Health Sciences at the University of Venda in Thohoyandou in South Africa.

\section{References}

1. Denno DM, Hoopes AJ, Chandra-Mouli V: Effective strategies to provide adolescent sexual and reproductive health services and to increase demand and community support. Journal of adolescent health 2015, 56(1):S22-S41.

2. Mantula F, Saloojee H: Child Sexual Abuse in Zimbabwe. Journal Of Child Sexual Abuse 2016, 25(8):866-880.

3. Mudonhi N, Nunu WN, Ndlovu B, Khumalo N, Dube O: Adolescents and Parents' Perceptions of Condom Distribution in Selected Secondary Schools in the High Density Suburbs of Bulawayo, Zimbabwe. Sexuality \& Culture 2019:1-19.

4. Hegamin-Younger C, Merrick J: Caribbean Adolescents : Misuse and Abuse of Alcohol. New York: Nova Science Publishers, Inc; 2017.

5. Ismail S, Shajahan A, Sathyanarayana Rao TS, Wylie K: Adolescent sex education in India: Current perspectives. Indian journal of psychiatry 2015, 57(4):333-337.

6. Darroch JE: Research gaps in adolescent sexual and reproductive health. In.: The Guttmacher Institute; 2016. 
7. Langhaug L, Cowan F, Nyamurera T, Power on Behalf Of The Regai Dzive Shiri Study Group R: Improving young people's access to reproductive health care in rural Zimbabwe. AIDS care 2003, 15(2):147-157.

8. Shumba K, Lubombo M: Cultural competence: a framework for promoting voluntary medical male circumcision among VaRemba communities in Zimbabwe. African journal of AIDS research : AJAR 2017, 16(2):165-173.

9. Greer S, Wismar M, Figueras J: Strengthening Health System Governance : Better Policies, Stronger Performance. New York, NY: McGraw-Hill Education; 2016.

10. Bassani C: Adolescent Behavior. New York: Nova Science Publishers, Inc; 2012.

11. Swartzendruber A, Zenilman JM: A national strategy to improve sexual health. JAMA 2010, 304(9):1005-1006.

12. Kinghorn G: A sexual health and HIV strategy for England: This ambitious strategy could, if properly resourced, greatly improve sexual health. In.: British Medical Journal Publishing Group; 2001.

13. Freedman L: Strategy : A History. Oxford: Oxford University Press; 2013.

14. Aggleton P, Campbell C: Working with young people-towards an agenda for sexual health. Sexual and Relationship Therapy 2000, 15(3):283-296.

15. Tofthagen R, Fagerstrøm LM: Rodgers' evolutionary concept analysis - a valid method for developing knowledge in nursing science R. Tofthagen, L.M. Fagerstrøm Presentation of Rodgers' evolutionary concept analysis. Scandinavian Journal of Caring Sciences 2010, 24:21-31.

16. Brilowski GA, Cecilia Wendler M: An evolutionary concept analysis of caring. Journal of advanced nursing 2005, 50(6):641-650.

17. Endacott R: Clarifying the concept of need: a comparison of two approaches to concept analysis. $J$ Adv Nurs 1997, 25(3):471-476.

18. Hui Liu D-LL, Irene Su: <strong>Editorial Comment of Walker and Avant's method of concept analysis</strong>. <span style='display:none'>3</span>TMR Integrative Nursing 2018, 2(1):1-3.

19. Kmet LM, Cook LS, Lee RC: Standard quality assessment criteria for evaluating primary research papers from a variety of fields. 2004.

20. Shea BJ, Grimshaw JM, Wells GA, Boers M, Andersson N, Hamel C, Porter AC, Tugwell P, Moher D, Bouter LM: Development of AMSTAR: a measurement tool to assess the methodological quality of systematic reviews. BMC medical research methodology 2007, 7(1):10.

21. Edwards WM, Coleman E: Defining sexual health: a descriptive overview. Archives of sexual Behavior 2004, 33(3):189-195.

22. Giami A: Sexual health: the emergence, development, and diversity of a concept. Annual review of sex research 2002, 13(1):1-35.

23. Mabvurira V, Motsi PD, Masuka T, Chigondo E: The "politics" of sexual identities in Zimbabwe: A Social Work perspective? 2012. 
24. Aarmo M: How homosexuality became 'un-African': The case of Zimbabwe. Female desires: Samesex relations and transgender practices across cultures 1999:255-280.

25. Elam G, Fenton KA: Researching sensitive issues and ethnicity: lessons from sexual health. Ethnicity and Health 2003, 8(1):15-27.

26. Obach A, Sadler M, Cabieses B: Intersectoral strategies between health and education for preventing adolescent pregnancy in Chile: Findings from a qualitative study. Health Expectations 2019, 22(2):183-192.

27. Cornelius JB, Appiah JA: Using mobile technology to promote safe sex and sexual health in adolescents: current practices and future recommendations. Adolescent Health, Medicine And Therapeutics 2016, 7:43-57.

28. Health MO: Sexual and Reproductive Health Needs Of Adolescents in Zimbabwe. International Perspectives on Sexual \& Reproductive Health 2014, 40(3):1-8.

29. Kirby D: The impact of schools and school programs upon adolescent sexual behavior. $J$ Sex Res 2002, 39(1):27-33.

30. Grunseit A, Kippax S: Impact of HIV and sexual health education on the sexual behaviour of young people: a review update. 1997.

31. Veale HJ, Sacks-Davis R, Weaver ER, Pedrana AE, Stoové MA, Hellard ME: The use of social networking platforms for sexual health promotion: identifying key strategies for successful user engagement. BMC public health 2015, 15(1):85.

32. Coe A-B, Goicolea I, Hurtig A-K, San Sebastian M: Understanding How Young People Do Activism: Youth Strategies on Sexual Health in Ecuador and Peru. Youth \& Society 2015, 47(1):3-28.

33. Khalesi ZB, Simbar M, Azin SA, Zayeri F: Public sexual health promotion interventions and strategies: A qualitative study. Electronic Physician 2016, 8(6):2489-2496.

34. Ndwiga C, Abuya T, Mutemwa R, Kimani JK, Colombini M, Mayhew S, Baird A, Wayua Muia R, Kivunaga $\mathrm{J}$, Warren $\mathrm{CE}$ : Exploring experiences in peer mentoring as a strategy for capacity building in sexual reproductive health and HIV service integration in Kenya. BMC Health Services Research 2014, 14(1):1-20.

35. Peña R, Quintanilla M, Navarro K, Martínez J, Castillo V, Pérez W, Källestál C: Evaluating a Peer Intervention Strategy for the Promotion of Sexual Health-Related Knowledge and Skills in 10- to 14year-old Girls. Findings from the "Entre Amigas" Project in Nicaragua. American Journal of Health Promotion 2008, 22(4):275-281.

36. Bishop E: Challenging homogenous representations of rural youth through a reconceptualisation of young rural Tasmanian's sexual health strategies. Health Sociology Review 2013, 22(2):124-136.

37. Gott M, Galena E, Hinchliff S, Elford H: "Opening a can of worms": GP and practice nurse barriers to talking about sexual health in primary care. Family practice 2004, 21(5):528-536.

38. Gott M, Hinchliff S, Galena E: General practitioner attitudes to discussing sexual health issues with older people. Social science \& medicine 2004, 58(11):2093-2103. 
39. Epprecht M: Sexual minorities, human rights and public health strategies in Africa. African Affairs 2012, 111(443):223-243.

40. Oakley A, Fullerton D, Holland J, Arnold S, Dawson MF, Kelley P, McGrellis S: Sexual health education interventions for young people: a methodological review. Bmj 1995, 310(6973):158-162.

41. Flicker S, Guta A: Ethical approaches to adolescent participation in sexual health research. Journal of Adolescent Health 2008, 42(1):3-10.

42. Bearinger $L H$, Sieving RE, Ferguson J, Sharma V: Global perspectives on the sexual and reproductive health of adolescents: patterns, prevention, and potential. The lancet 2007, 369(9568):1220-1231.

43. Anike U, Govender I, Ndimande JV, Tumbo J: Complications of traditional circumcision amongst young Xhosa males seen at St Lucy's Hospital, Tsolo, Eastern Cape, South Africa. African Journal of Primary Health Care \& Family Medicine 2013, 5(1):488.

44. Biddlecom AE, Munthali A, Singh S, Woog V: Adolescents' views of and preferences for sexual and reproductive health services in Burkina Faso, Ghana, Malawi and Uganda. African journal of reproductive health 2007, 11(3):99.

45. Ngwenya S: Communication of reproductive health information to the rural girl child in Filabusi, Zimbabwe: Afr Health Sci. 2016 Jun;16(2):451-61. doi:10.4314/ahs.v16i2.13.

46. Mpondo F, Ruiter RAC, Schaafsma D, van den Borne B, Reddy PS: Understanding the role played by parents, culture and the school curriculum in socializing young women on sexual health issues in rural South African communities. SAHARA J: Journal Of Social Aspects Of HIV/AIDS Research Alliance 2018, 15(1):42-49.

47. Lebese RT, Davhana-Maselesele M, Obi CL: Sexual health dialogue between parents and teenagers: an imperative in the HIV/AIDS era. Curationis 2010, 33(3):33-42.

\section{Tables}

Table 1: Summary of key Attributes of HSSs in relation to ASH

\begin{tabular}{|l|l|}
\hline Key Attribute Factors & Supporting Literature \\
\hline Contextual & {$[5,14,21,25,27-29]$} \\
\hline Dynamic & {$[14,21,22,26-29]$} \\
\hline Activism and advocacy & {$[30-35]$} \\
\hline Inefficient & {$[14,21,25,26,36-38]$} \\
\hline
\end{tabular}

Table 2: Summary of key Antecedents of HSSs in relation to ASH 


\begin{tabular}{|l|l|}
\hline Key Antecedent Factors & Supporting Literature \\
\hline Adolescent sexual rights & {$[2,14,31,39-41]$} \\
\hline Lack of understanding of what sexual health is & {$[14,21,37,38,40]$} \\
\hline Need for Integrated ASH Systems & {$[5,11,40,42]$} \\
\hline Available Resources & {$[37,38]$} \\
\hline Type of society & {$[14,25,26,36,37,43]$} \\
\hline
\end{tabular}

Table 3: Summary of key Consequences of HSSs on ASH

\begin{tabular}{|l|l|}
\hline Key Consequences Factors & Supporting Literature \\
\hline Weak adolescent programs constrained by different factors & {$[2,5]$} \\
\hline Parallel or Fragmented ASH Systems resulting in low impact & {$[11]$} \\
\hline Vulnerability and heightened risks for poor health outcomes & {$[3,5,27-29,42]$} \\
\hline Challenges in interacting with different ethnic groups and gender & {$[23,24,37,38]$} \\
\hline
\end{tabular}

Table 4: AMSTAR tool results on Methodological Quality 


\begin{tabular}{|c|c|c|}
\hline AMSTAR criteria / Question & Response & Justification \\
\hline $\begin{array}{l}\text { 1. Was an 'a priori' design provided? } \\
\text { The research question and inclusion criteria should be } \\
\text { established before the conduct of the review. }\end{array}$ & Yes & $\begin{array}{l}\text { A review } \\
\text { protocol was } \\
\text { developed to } \\
\text { prior } \\
\text { conducting the } \\
\text { literature } \\
\text { review }\end{array}$ \\
\hline $\begin{array}{l}\text { 2. Was there duplicate study selection and data extraction? } \\
\text { There should be at least two independent data extractors and } \\
\text { a consensus procedure for disagreements should be in place. }\end{array}$ & Yes & $\begin{array}{l}\text { Three } \\
\text { reviewers } \\
\text { reviewed the } \\
\text { articles and } \\
\text { reports and a } \\
\text { clear } \\
\text { procedure } \\
\text { describe to } \\
\text { iron on } \\
\text { disagreements } \\
\text { and reach } \\
\text { consensus }\end{array}$ \\
\hline $\begin{array}{l}\text { 3. Was a comprehensive literature search performed? } \\
\text { At least two electronic sources should be searched. The } \\
\text { report must include years and databases used (e.g. Central, } \\
\text { EMBASE, and MEDLINE). Key words and / or MESH terms } \\
\text { must be stated and where feasible the search strategy should } \\
\text { be provided. All searches should be supplemented by } \\
\text { consulting current contents, reviews, textbooks, specialized } \\
\text { registers, or experts in the particular field of study, and by } \\
\text { reviewing the references in the studies found. }\end{array}$ & Yes & $\begin{array}{l}\text { The review } \\
\text { focussed on } \\
\text { original } \\
\text { quantitative } \\
\text { and qualitative } \\
\text { research and } \\
\text { reports } \\
\text { obtained from } \\
\text { Google } \\
\text { Scholar, } \\
\text { PUBMED, } \\
\text { EBSCO and } \\
\text { Science } \\
\text { Direct. }\end{array}$ \\
\hline $\begin{array}{l}\text { 4. Was the status of publication (i.e. grey literature) used as } \\
\text { an inclusion criterion? } \\
\text { The authors should state that they searched for reports } \\
\text { regardless of their publication type. The authors should state } \\
\text { whether or not they excluded any reports (from the } \\
\text { systematic review), based on their publication status, } \\
\text { language etc. }\end{array}$ & Yes & $\begin{array}{l}\text { The inclusion } \\
\text { and exclusion } \\
\text { criteria is fully } \\
\text { described and } \\
\text { also } \\
\text { summarised in } \\
\text { the PRISMA } \\
\text { flow diagram } \\
\text { presented in } \\
\text { this review as } \\
\text { Fig } 1 \text {. }\end{array}$ \\
\hline $\begin{array}{l}\text { 5. Was a list of studies (included and excluded) provided? } \\
\text { A list of included and excluded studies should be provided. }\end{array}$ & No & $\begin{array}{l}\text { The list was } \\
\text { not provided } \\
\text { but can be } \\
\text { supplied upon } \\
\text { request }\end{array}$ \\
\hline 6. Were the characteristics of the included studies provided? & Yes & $\begin{array}{l}\text { These were } \\
\text { summarised }\end{array}$ \\
\hline
\end{tabular}


In an aggregated form such as a table, data from the original studies should be provided on the participants, interventions and outcomes. The ranges of characteristics in all the studies analyzed e.g. age, race, sex, relevant socioeconomic data, disease status, duration, severity, or other diseases should be reported.
7. Was the scientific quality of the included studies assessed and documented?

'A priori' methods of assessment should be provided (e.g., for effectiveness studies if the author(s) chose to include only randomized, double-blind, placebo controlled studies, or allocation concealment as inclusion criteria); for other types of studies alternative items will be relevant. as Tables

that

summarised

contributions

of literature

according to

three Rodgers

characteristics

that

attributes,

antecedents

and

consequences.

Yes

These were assessed using the 14 point quality assessment tool and

Rodgers Evolutionary Conceptual Framework.

8. Was the scientific quality of the included studies used Yes appropriately in formulating conclusions?

The results of the methodological rigor and scientific quality should be considered in the analysis and the conclusions of the review, and explicitly stated in formulating recommendations.

\section{Were the methods used to combine the findings of studies appropriate?}

For the pooled results, a test should be done to ensure the studies were combinable, to assess their homogeneity (i.e. Chi squared test for homogeneity, I2). If heterogeneity exists a random effects model should be used and/or the clinical appropriateness of combining should be taken into consideration (i.e. is it sensible to combine?).

10. Was the likelihood of publication bias assessed?

An assessment of publication bias should include a combination of graphical aids (e.g., funnel plot, other available tests) and / or statistical tests (e.g., Egger regression test).
Studies that were included were guided and met

Rodgers

Evolutionary conceptual analysis framework requirements

N/A

The review had a guiding conceptual framework, therefore the combination of articles were guided by framework

The review was guided by Rodgers' Evolutionary Concept Analysis framework. However limitations of this systematic 


\begin{tabular}{|l|l|l|} 
& $\begin{array}{l}\text { review are } \\
\text { presented. }\end{array}$ \\
\hline $\begin{array}{l}\text { 11. Was the conflict of interest stated? } \\
\text { Potential sources of support should be clearly acknowledged } \\
\text { in both the systematic review and the included studies. }\end{array}$ & $\begin{array}{l}\text { All authors } \\
\text { declared that } \\
\text { they did not } \\
\text { have any } \\
\text { conflict of } \\
\text { interest. }\end{array}$ \\
\hline
\end{tabular}

\section{Figures}




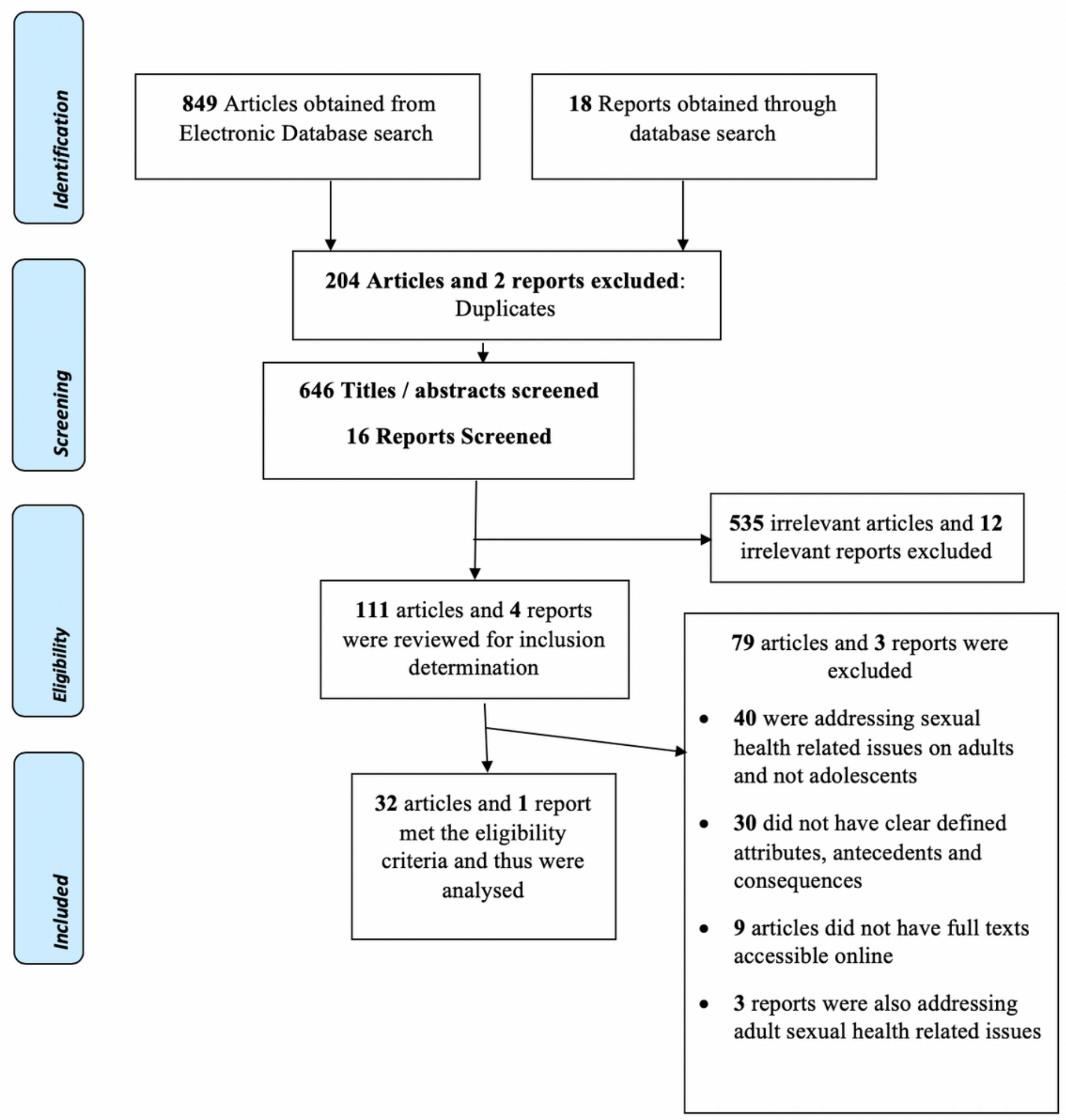

Figure 1

PRISMA Flow Diagram 


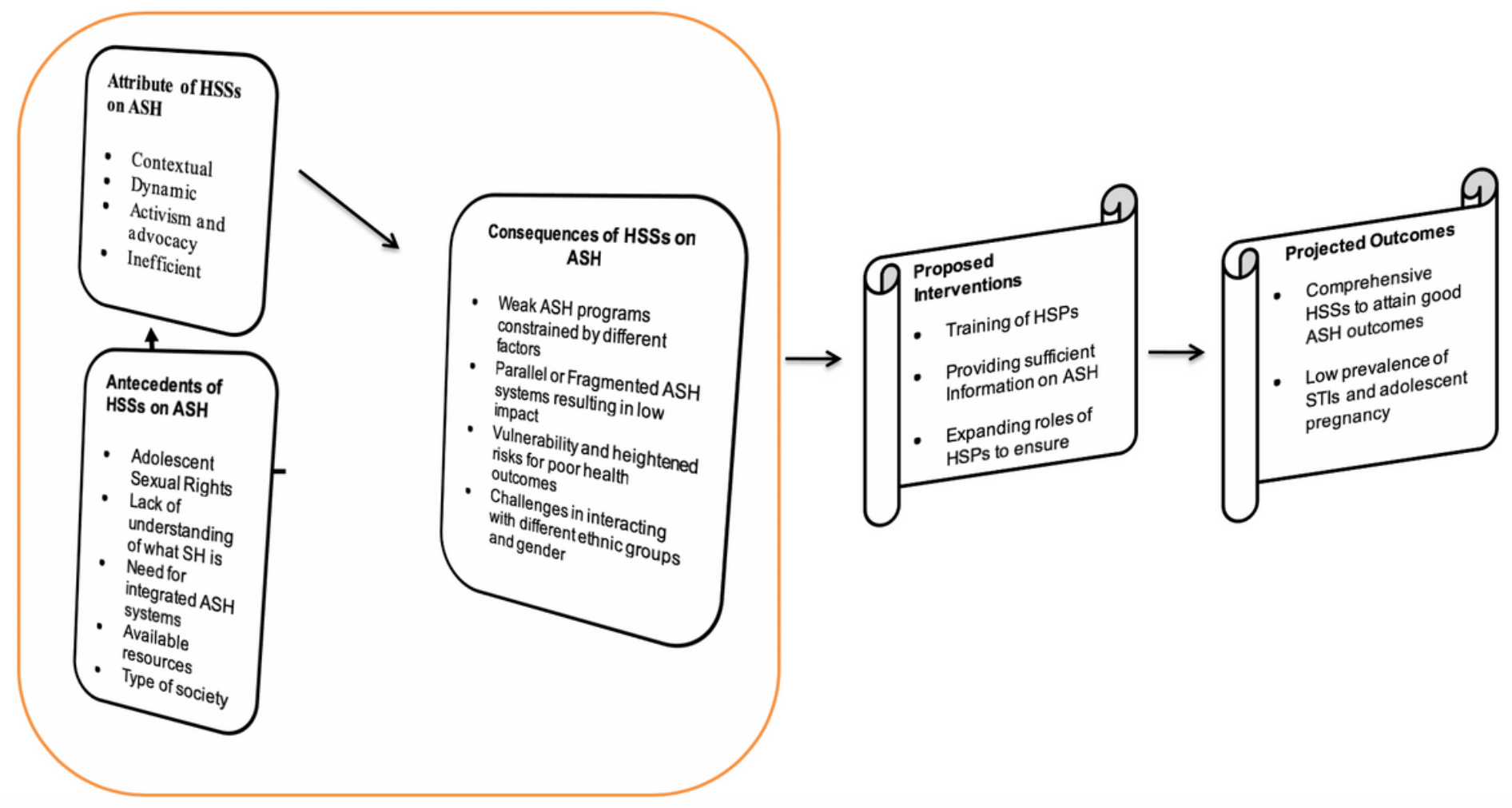

Figure 2

CF of HSSs and ASH as informed by findings. 